\title{
Effect of sintering temperature on thermoelectric properties of CdO ceramics
}

\author{
Xinran ZHANG, Huiling LI, Jianglong WANG ${ }^{*}$ \\ Hebei Key Lab of Optic-electronic Information and Materials, College of Physics Science and Technology, \\ Hebei University, Baoding 071002, China
}

Received: February 01, 2015; Revised: April 30, 2015; Accepted: May 03, 2015

(C) The Author(s) 2015. This article is published with open access at Springerlink.com

\begin{abstract}
The effect of sintering temperature on thermoelectric properties of $\mathrm{CdO}$ ceramics was investigated by solid-state reaction method within the temperature range of $700-1000{ }^{\circ} \mathrm{C}$. With the increase of sintering temperature, both the grain size and the carrier concentration of these samples increased, while the Seebeck coefficient decreased. The highest dimensionless figure of merit $Z T, 0.34$, was obtained at $1023 \mathrm{~K}$ for the sample sintered at $800{ }^{\circ} \mathrm{C}$, suggesting the potential application of $\mathrm{CdO}$ ceramics in thermoelectric (TE) devices.
\end{abstract}

Keywords: thermoelectric (TE); CdO ceramics; sintering temperature; solid-state reaction

\section{Introduction}

Thermoelectric (TE) materials can directly convert heat into electricity through Seebeck effect and electricity into heat through Peltier effect, and therefore they have promising applications in power generation and cooling. The performance of a TE material is characterized by the dimensionless figure of merit, $Z T=\left(S^{2} \sigma / \kappa\right) T$, where $S, \sigma, \kappa$, and $T$ are the Seebeck coefficient, electrical conductivity, thermal conductivity, and absolute temperature, respectively. The conventional thermoelectric materials are mainly intermetallic compounds and alloys. However, due to surface oxidation and sublimation at high temperature and toxic or scarce elements contained, the practical application of these materials is limited. Oxides can overcome these problems and therefore have attracted increasing attentions to the TE community, particularly to those

\footnotetext{
* Corresponding author.

E-mail: jlwang@hbu.edu.cn
}

who are interested in high temperature applications in air [1-15]. Transparent conductive oxides (TCOs), mainly including $\mathrm{ZnO}$-, $\mathrm{In}_{2} \mathrm{O}_{3^{-}}, \mathrm{SnO}_{2}$-, and $\mathrm{TiO}_{2}$-based oxides, have been extensively studied as a promising class of n-type TE materials for high temperature applications because of their low electrical resistivity and good stability at high temperature in air [9-15]. So far, the best $Z T$ for above TCOs-based TE materials has been achieved in $\mathrm{Al}$ and $\mathrm{Ga}$ dually doped $\mathrm{ZnO}$ ceramics [10].

Cadmium oxide $(\mathrm{CdO})$ is an n-type TCO with high carrier mobility. It has a simple cubic rock-salt crystal structure with direct band gap of $2.28 \mathrm{eV}$ and indirect band gap of $0.84 \mathrm{eV}$ [16]. Nonstoichiometric $\mathrm{CdO}$ usually exhibits low electrical resistivity due to native defects of oxygen vacancies and $\mathrm{Cd}$ interstitials [17]. Compared with $\mathrm{ZnO}, \mathrm{CdO}$ has heavier constituting elements and lower Debye temperature $\left(\theta_{\mathrm{D}} \approx 255 \mathrm{~K}\right)$, and hence a relative lower phonon thermal conductivity [18]. These features suggest $\mathrm{CdO}$ might be a potential novel n-type oxide TE material despite its drawbacks of 
toxicity and volatility at high temperature. Very recently, a good TE performance has been demonstrated in $\mathrm{CdO}$, and the effect of rare-earth elements doping on the TE properties of $\mathrm{CdO}$ ceramics has been investigated by our research group [19-21]. It is known that the carrier concentration and microstructure can greatly influence the carrier and phonon transport properties in TE materials, which are directly related with these three TE parameters, $S, \sigma$, and $\kappa$. Therefore, one common approach to improve the performance of a TE material is tuning its carrier concentration and microstructure. In this paper, the carrier concentration and microstructure of $\mathrm{CdO}$ ceramics were tuned via changing the sintering temperature of the samples, and the influence of sintering temperature on the thermoelectric properties of $\mathrm{CdO}$ ceramics was investigated. It was found that both the electrical resistivity and the Seebeck coefficient decrease while the thermal conductivity increases with increasing sintering temperature. We obtained a high $Z T$ of 0.34 at $1023 \mathrm{~K}$ for the sample sintered at $800{ }^{\circ} \mathrm{C}$. This value is comparable to that reported for the best n-type oxide ceramic thermoelectric materials.

\section{Experimental}

Polycrystalline $\mathrm{CdO}$ ceramic samples were prepared by the traditional solid-state reaction sintering method with the commercial high purity $\mathrm{CdO}$ powders $(99.95 \%$, Alfa Aesar) as the starting materials. After fully grinding, the powders of $\mathrm{CdO}$ were first presintered at $600{ }^{\circ} \mathrm{C}$ for about $3 \mathrm{~h}$ to decarbonate and then were compacted in pellets at $10 \mathrm{MPa}$. Finally, these pellets were sintered in air at temperatures of 700, 800, 900, and $1000{ }^{\circ} \mathrm{C}$ for about $20 \mathrm{~h}$.

The crystal structure of the sintered $\mathrm{CdO}$ ceramics was examined by X-ray diffraction (XRD) with a Bruker D8 Advance X-ray powder diffractometer. The microstructure was measured using a Philips XL-30S scanning electron microscope (SEM). The room temperature carrier concentration $n$ and mobility $\mu$ were determined by using the van der Pauw method with a Hall measurement system (ET-900, China). The electrical resistivity $\rho$ and the Seebeck coefficient $S$ were simultaneously measured by the standard DC four-probe technique with an LSR-3 measurement system (Linseis, Germany) from 300 to $1023 \mathrm{~K}$ under the low pressure helium atmosphere. The thermal conductivity $\kappa$ was calculated from the specific heat capacity $C_{P}$, thermal diffusivity $D$, and density $d$ according to the equation $\kappa=D C_{P} d$, where $D$ was measured using the laser flash technique with LFA1000 system (Linseis, Germany); $C_{P}$ was measured by a differential scanning calorimeter (Netzsch DSC200F3); and $d$ was determined by the Archimedes method. The density of $\mathrm{CdO}$ ceramics was found to increase with the sintering temperature and was about 7.81, 8.09, 8.16, and $8.22 \mathrm{~g} / \mathrm{cm}^{3}$ for samples sintered at $700,800,900$, and $1000{ }^{\circ} \mathrm{C}$, respectively.

\section{Results and discussion}

Figure 1 presents the XRD $\theta-2 \theta$ scans of these four samples sintered at different temperatures. All peaks in the XRD pattern of each sample can be indexed to $\mathrm{CdO}$ with space group $F m \overline{3} m$ (PDF \#780653). With increasing the sintering temperature, the width of XRD peaks becomes slightly narrower and the intensity of the peaks becomes stronger (as seen in the inset of Fig. 1), indicating that both the crystalline quality and grain size of the samples are increased at higher sintering temperatures. Figure 2 shows the SEM images from the fractured cross-sections of these four samples. It can be clearly seen that the average grain size of the samples becomes larger as the sintering temperature increases, which is about $1,5,10$, and $15 \mu \mathrm{m}$ for samples sintered at $700,800,900$, and $1000{ }^{\circ} \mathrm{C}$, respectively.

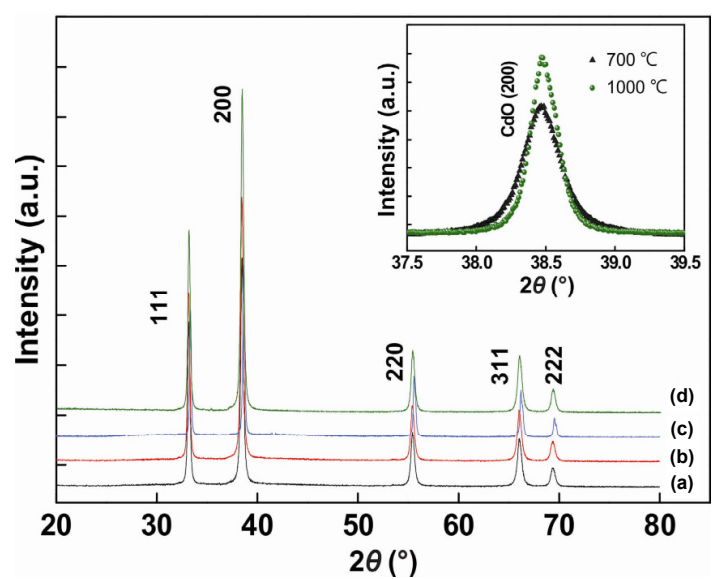

Fig. 1 XRD $\theta-2 \theta$ patterns of $\mathrm{CdO}$ ceramics sintered at different temperatures of (a) $700{ }^{\circ} \mathrm{C}$, (b) $800{ }^{\circ} \mathrm{C}$, (c) $900{ }^{\circ} \mathrm{C}$, and (d) $1000{ }^{\circ} \mathrm{C}$. The inset is the magnified curves of $\mathrm{CdO}$ (200) peak for samples sintered at $700{ }^{\circ} \mathrm{C}$ and $1000{ }^{\circ} \mathrm{C}$. 


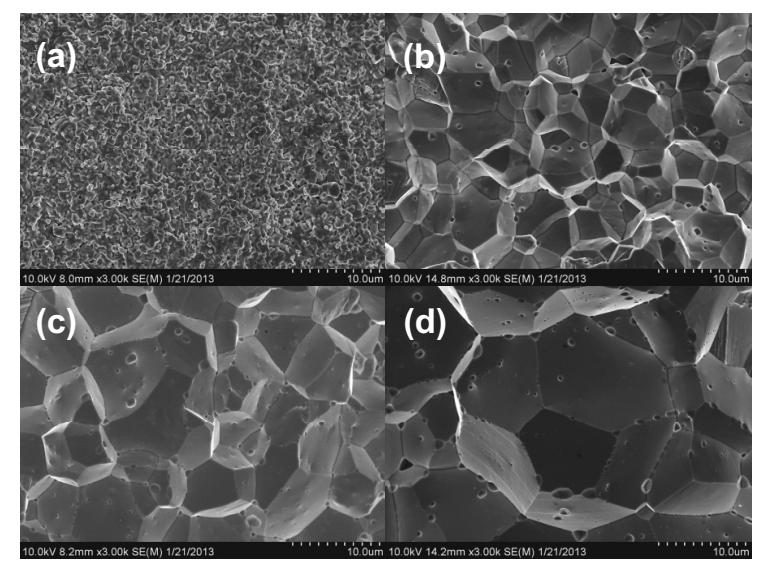

Fig. 2 SEM images of $\mathrm{CdO}$ ceramics sintered at different temperatures of (a) $700{ }^{\circ} \mathrm{C}$, (b) $800{ }^{\circ} \mathrm{C}$, (c) $900{ }^{\circ} \mathrm{C}$, and (d) $1000{ }^{\circ} \mathrm{C}$.

Figure 3 presents the room temperature carrier concentration $n$ and mobility $\mu$ of these $\mathrm{CdO}$ ceramic samples sintered at different temperatures. With the increase of sintering temperature, $n$ increases while $\mu$ decreases. The increase in the carrier concentration $n$ with the sintering temperature is suggested to be caused by the increase of oxygen vacancies or cadmium interstitials. At higher sintering temperatures, more defects are thermally generated, resulting in more oxygen vacancies or cadmium interstitials. The variation of mobility $\mu$ with the sintering temperature can be explained by considering the following competing factors: (1) as the sintering temperature increases, both the bulk density and the grain size of the samples increase, increasing the time between electron scattering events of charge carriers at grain boundaries and thus increasing the mobility $\mu[22,23]$; (2) the increase of sintering temperature leads to an increase in carrier concentration $n$, resulting in a decrease in

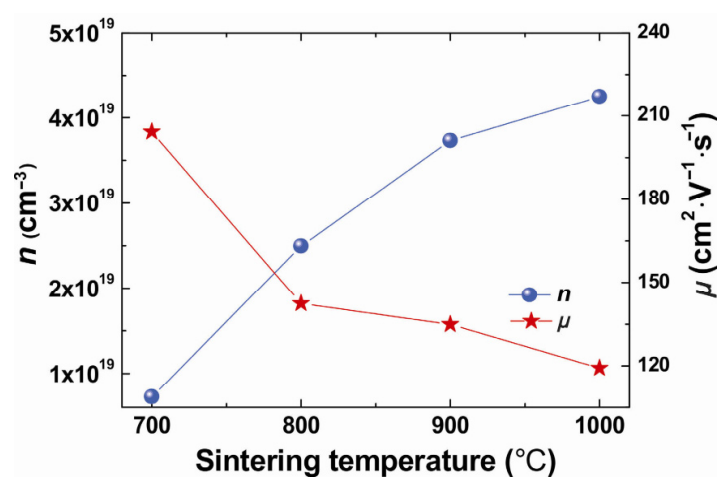

Fig. 3 Room temperature carrier concentration $n$ and Hall mobility $\mu$ of $\mathrm{CdO}$ ceramics sintered at different temperatures. mobility $\mu$ due to the enhanced scattering of carriers by more ionized impurities. From the above competing factors, it is suggested that the reduction in the mobility $\mu$ with the sintering temperature is mainly due to the ionized impurity scattering for these $\mathrm{CdO}$ ceramic samples [24].

Figure 4(a) displays the temperature dependence of electrical resistivity $\rho$ for these four $\mathrm{CdO}$ ceramic samples. The room temperature electrical resistivity exhibits a rapid decrease when the sintering temperature increases from 700 to $900{ }^{\circ} \mathrm{C}$, and further increasing the sintering temperature only results in a slight decrease in $\rho$. The decrease of $\rho$ with the sintering temperature is mainly due to the increase of carrier concentration $n$ since the mobility $\mu$ decreases at higher sintering temperatures. Moreover, Fig. 4(a) shows that the electrical resistivity of the samples sintered at $700{ }^{\circ} \mathrm{C}$ and $800{ }^{\circ} \mathrm{C}$ first increases with the temperature, reaches the maximum value at a certain temperature $T^{*}$, and then decreases. It should be mentioned here that $T^{*}$ will be shifted to higher temperatures as the carrier concentration $n$ is increased, and the $T^{*}$ value for samples sintered at $900{ }^{\circ} \mathrm{C}$ and $1000{ }^{\circ} \mathrm{C}$ is out of our temperature measurement range. The above $\rho-T$ behavior has been observed in many other semiconductors and can be explained in the following manner. In extrinsic temperature regime of a semiconductor $\left(T<T^{*}\right)$, the number of thermally excited charge carriers is very small and the resistivity is primarily determined by the extrinsic carriers. As the temperature increases, the thermally excited intrinsic carriers across the energy gap of the semiconductor can not be ignored and thus begin to contribute to the resistivity, so the resistivity will decrease when the temperature is higher than $T^{*}$ [25].

The temperature variation of the Seebeck coefficient $S$ for these four CdO ceramic samples is shown in Fig. 4(b). The negative $S$ values indicate the n-type conduction in these samples. The absolute value of the Seebeck coefficient, $|S|$, for all samples increases with the temperature $T$ when $T$ is lower than $T^{*}$, which is consistent with the characteristic behavior of an n-type degenerate semiconductor. The reduction of $|S|$ under the condition of $T>T^{*}$ is caused by the contribution of thermally excited charge carriers as we described above. In addition, Fig. 4(b) shows $|S|$ decreases with the increase of sintering temperature. The variation of $|S|$ of $\mathrm{CdO}$ ceramics with sintering temperature can be simply explained as follows. In the single parabolic band model, 

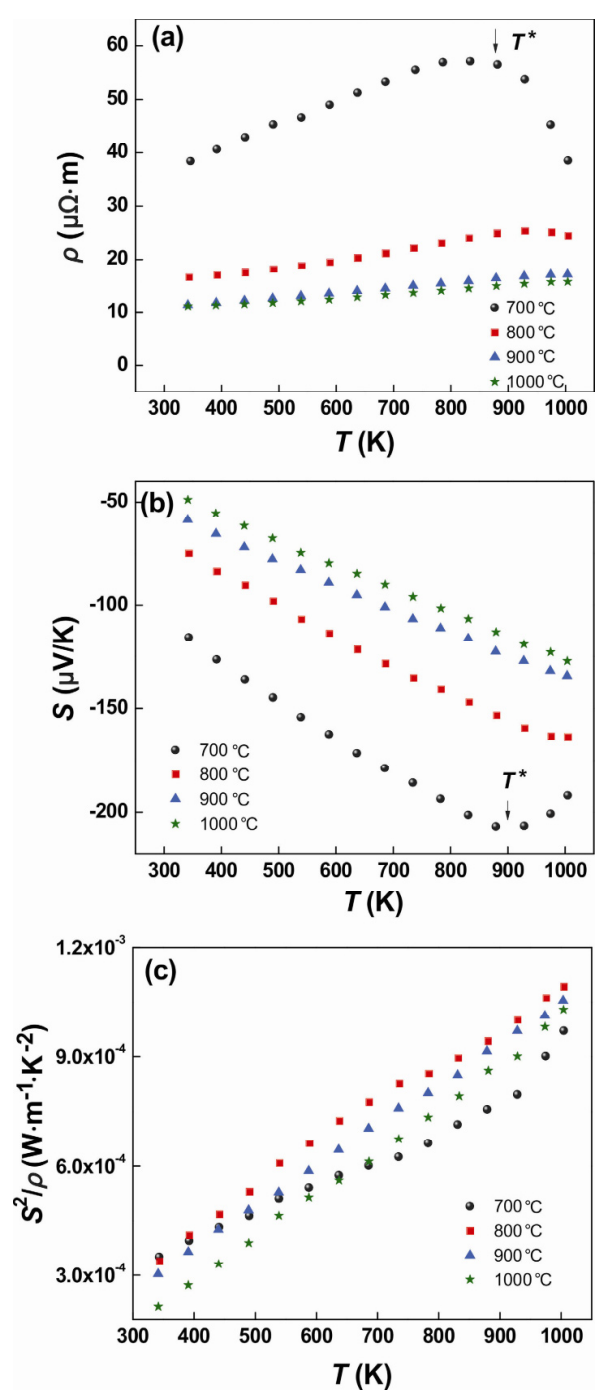

Fig. 4 Temperature dependence of (a) electrical resistivity $\rho$, (b) Seebeck coefficient $S$, and (c) power factor $S^{2} / \rho$ for $\mathrm{CdO}$ ceramics sintered at different temperatures.

the absolute value $|S|$ of an n-type degenerate semiconductor can be expressed by [26]

$$
|S|=\frac{8 \pi^{2} k_{\mathrm{B}}^{2}}{3 e h^{2}}\left(\frac{\pi}{3 n}\right)^{2 / 3} m^{*} T
$$

where $k_{\mathrm{B}}, h$, and $m^{*}$ are the Boltzmann constant, Planck constant, and carrier effective mass, respectively. With the increase of sintering temperature, the carrier concentration $n$ will increase and thus the $|S|$ will decrease according to Eq. (1). Based on the measured carrier concentration and the Seebeck coefficient at room temperature, the effective mass can be estimated from Eq. (1), which is $0.126,0.185,0.190$, and 0.173 (in unit of free electron mass $m_{0}$ ) for samples sintered at $700{ }^{\circ} \mathrm{C}, 800{ }^{\circ} \mathrm{C}, 900{ }^{\circ} \mathrm{C}$, and $1000{ }^{\circ} \mathrm{C}$, respectively. It can be seen that the effective mass of $\mathrm{CdO}$ samples sintered at $700{ }^{\circ} \mathrm{C}, 800{ }^{\circ} \mathrm{C}$, and $900{ }^{\circ} \mathrm{C}$ increases with increasing carrier concentration, which is in agreement with the report from optical measurement [27]. The inconsistence for the $1000{ }^{\circ} \mathrm{C}$ sample might be due to the missed factors involving the non-parabolic band effect and the detailed scattering mechanism in Eq. (1). Figure 4(c) is the temperature dependence of the power factor $S^{2} / \rho$ for these four $\mathrm{CdO}$ ceramic samples, and the power factor $S^{2} / \rho$ of each sample is calculated with the corresponding $\rho-T$ and $S-T$ data shown in Fig. 4. The power factor of all samples increases with the increase of temperature. The sample sintered at $800{ }^{\circ} \mathrm{C}$ has the highest power factor of $1.1 \times 10^{-3} \mathrm{~W} \cdot \mathrm{m}^{-1} \cdot \mathrm{K}^{-2}$ at $1023 \mathrm{~K}$, which is comparable to that reported for other TCOs-based TE materials such as $\mathrm{ZnO}$ and $\mathrm{In}_{2} \mathrm{O}_{3}$ [13-15].

Figure 5(a) presents the thermal conductivity $\kappa$ as a function of temperature for these four $\mathrm{CdO}$ samples. With the increase of sintering temperature, the thermal conductivity of the samples $\kappa$ increases. To well understand this dependence of thermal conductivity on sintering temperature, we estimated the carrier thermal conductivity $\kappa_{\mathrm{e}}$ and the phonon thermal conductivity $\kappa_{\mathrm{ph}}$ of these four samples, which are displayed in Figs. 5(b) and 5(c), respectively. $\kappa_{\mathrm{e}}$ was estimated by the Wiedemann-Franz law $\kappa_{\mathrm{e}}=L T \sigma$, and $\kappa_{\mathrm{ph}}$ was calculated from the equation $\kappa_{\mathrm{ph}}=\kappa-\kappa_{\mathrm{e}}$.

Here, the Lorentz number $L$ of these our $\mathrm{CdO}$ samples at different temperatures is obtained by the calculations according to Ref. [28]. It can be seen that the carrier thermal conductivity $\kappa_{\mathrm{e}}$ of the samples increases with the sintering temperature because of the improvement of electrical conductivity at higher sintered temperatures. At the same time, the phonon thermal conductivity $\kappa_{\mathrm{ph}}$ of the samples is also found to increase with increasing the sintering temperature. As the sintering temperature increases, the grain size of the samples will become larger and thus the density of grain boundaries will decrease. Moreover, the increase of sintering temperature also results in a reduction in the porosity of the samples. In both cases, the phonon scattering will be suppressed, leading to an increase in $\kappa_{\mathrm{ph}}$.

We calculated the $Z T$ values of these samples, which are shown in Fig. 6. The sample sintered at $800{ }^{\circ} \mathrm{C}$ exhibits the best TE performance in the whole temperature range and its $Z T$ reaches 0.34 at $1023 \mathrm{~K}$. 

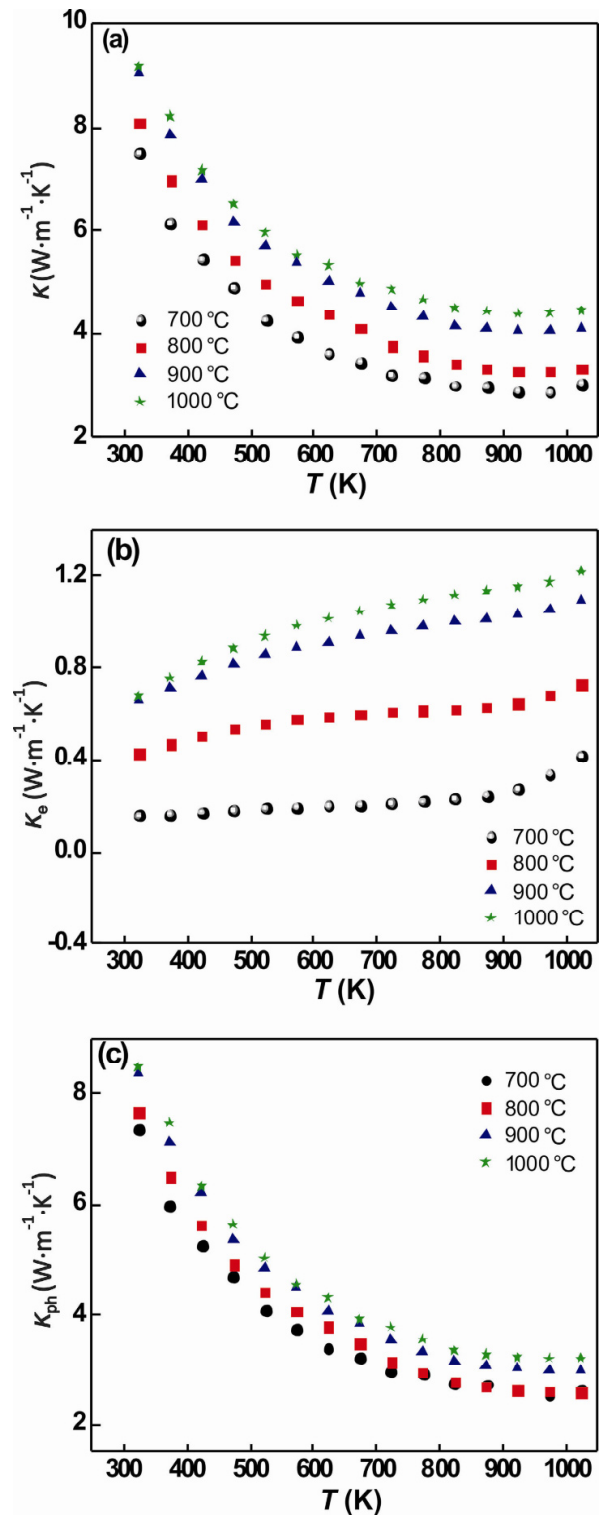

Fig. 5 Temperature dependence of (a) thermal conductivity $\kappa$, (b) carrier thermal conductivity $\kappa_{\mathrm{e}}$, and (c) phonon thermal conductivity $\kappa_{\mathrm{ph}}$ for $\mathrm{CdO}$ ceramics sintered at different temperatures.

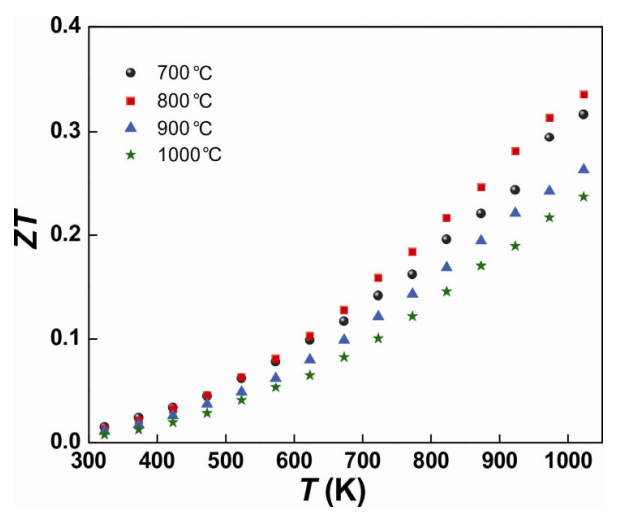

Fig. 6 Temperature dependence of $Z T$ for $\mathrm{CdO}$ ceramics sintered at different temperatures.
This value is comparable to that reported for the best n-type oxide ceramic TE materials $[7,11,15,16]$, suggesting the potential applications of $\mathrm{CdO}$ in $\mathrm{TE}$ devices. Further improvement in $Z T$ is possible by nano-engineering approach.

\section{Conclusions}

$\mathrm{CdO}$ ceramics were synthesized by the solid-state reaction sintering method, and the effect of sintering temperature on their thermoelectric properties was investigated. With the increase of sintering temperature, both the electrical resistivity and Seebeck coefficient of these samples decreased while the thermal conductivity increased. Detailed mechanisms were proposed to explain the experimental results. The sample sintered at $800{ }^{\circ} \mathrm{C}$ exhibited the best TE performance in the whole temperature range, and its $Z T$ reached 0.34 at $1023 \mathrm{~K}$. Our works demonstrated that the sintered $\mathrm{CdO}$ ceramics have potential application in oxide TE devices and their TE performance can be tuned by sintering temperature.

\section{Acknowledgements}

This work was supported by the Natural Science Foundation of Hebei Province, China (Grant No. A2014201176) and the Science and Technology Research Projects of Colleges and Universities in Hebei Province, China (Grant No. ZD2014018).

Open Access: This article is distributed under the terms of the Creative Commons Attribution License which permits any use, distribution, and reproduction in any medium, provided the original author(s) and the source are credited.

\section{References}

[1] Koumoto K, Wang Y, Zhang R, et al. Oxide thermoelectric materials: A nanostructuring approach. Annu Rev Mater Res 2010, 40: 363-394.

[2] Fergus JW. Oxide materials for high temperature thermoelectric energy conversion. J Eur Ceram Soc 2012, 32: $525-540$.

[3] Terasaki I, Sasago Y, Uchinokura K. Large thermoelectric power in $\mathrm{NaCO}_{2} \mathrm{O}_{4}$ single crystals. Phys Rev $B$ 1997, 56: R12658.

[4] Yin LH, Ang R, Huang ZH, et al. Exotic reinforcement of thermoelectric power driven by $\mathrm{Ca}$ doping in layered $\mathrm{Bi}_{2} \mathrm{Sr}_{2-x} \mathrm{Ca}_{x} \mathrm{Co}_{2} \mathrm{O}_{y}$. Appl Phys Lett 2013, 102: 141907. 
[5] Ohta H, Kim S, Mune Y, et al. Giant thermoelectric Seebeck coefficient of a two-dimensional electron gas in $\mathrm{SrTiO}_{3}$. Nat Mater 2007, 6: 129-134.

[6] Wang HC, Wang CL, Su WB, et al. Doping effect of La and Dy on thermoelectric properties of $\mathrm{SrTiO}_{3} . J \mathrm{Am}$ Ceram Soc 2011, 94: 838-842.

[7] Lee S, Bock JA, Trolier-McKinstry S, et al. Ferroelectric-thermoelectricity and Mott transition of ferroelectric oxides with high electronic conductivity. J Eur Ceram Soc 2012, 32: 3971-3988.

[8] Liu Y, Zhao L-D, Liu Y, et al. Remarkable enhancement in thermoelectric performance of $\mathrm{BiCuSeO}$ by $\mathrm{Cu}$ deficiencies. J Am Chem Soc 2012, 134: 3312.

[9] Kitagawa H, Kunisada T, Yamada Y, et al. Effect of boron-doping on thermoelectric properties of rutile-type titanium dioxide sintered materials. J Alloys Compd 2010, 508: 582-586.

[10] Ohtaki M, Araki K, Yamamoto K. High thermoelectric performance of dually doped $\mathrm{ZnO}$ ceramics. J Electron Mater 2009, 38: 1234-1238.

[11] Teranishi T, Mori Y, Hayashi H, et al. Thermoelectric property of polycrystalline aluminum-doped zinc oxide enhanced by micropore foaming. J Am Ceram Soc 2012, 95: 690-695.

[12] Jood P, Mehta RJ, Zhang Y, et al. Al-doped zinc oxide nanocomposites with enhanced thermoelectric properties. Nano Lett 2011, 11: 4337-4342.

[13] Park K, Seong JK, Kim GH. NiO added $\mathrm{Zn}_{1-x} \mathrm{Ni}_{x} \mathrm{O}(0 \leqslant x$ $\leqslant 0.05)$ for thermoelectric power generation. $J$ Alloys Compd 2009, 473: 423-427.

[14] Bérardan D, Guilmeau E, Maignan A, et al. $\operatorname{In}_{2} \mathrm{O}_{3}$ :Ge, a promising n-type themoelectric oxide composite. Solid State Commun 2008, 146: 97-101.

[15] Liu Y, Lin Y-H, Lan J, et al. Effect of transition-metal cobalt doping on the thermoelectric performance of $\operatorname{In}_{2} \mathrm{O}_{3}$ ceramics. J Am Ceram Soc 2010, 93: 2938-2941.

[16] Koffyberg FP. Thermoreflectance spectra of CdO: Band gaps and band-population effects. Phys Rev B 1976, 13: 4470.

[17] Koffyberg FP. Electron concentration and mobility in semimetallic CdO. Can J Phys 1971, 49: 435-440.

[18] Madelung O, Rössler U, Schulz M. II-VI and I-VII Compounds; Semimagnetic Compounds. Berlin Heidelberg: Springer-Verlag, 1999, 41B: 1-5.

[19] Wang S, Liu F, Lü Q, et al. The effect of $\mathrm{Er}^{3+}$ doping on the structure and thermoelectric properties of $\mathrm{CdO}$ ceramics. J Eur Ceram Soc 2013, 33: 1763-1768.

[20] Wang S, Lü Q, Li L, et al. High-temperature thermoelectric properties of $\mathrm{Cd}_{1-x} \mathrm{Pr}_{x} \mathrm{O}$ ceramics. Scripta Mater 2013, 69: 533-536.

[21] Lü Q, Wang SF, Li LJ, et al. Electrical and thermal transport properties of $\mathrm{CdO}$ ceramics. Science China Physics, Mechanics \& Astronomy 2014, 57: 1644-1647.

[22] Park K, Ko KY, Kim J-G, et al. Microstructure and high-temperature thermoelectric properties of $\mathrm{CuO}$ and $\mathrm{NiO}$ co-substituted $\mathrm{NaCo}_{2} \mathrm{O}_{4}$. Mat Sci Eng B 2006, 129: 200-206.

[23] Park K, Kim KK, Seong JK, et al. Improved thermoelectric properties by adding $\mathrm{Al}$ for $\mathrm{Zn}$ in $(\mathrm{ZnO})_{m} \mathrm{In}_{2} \mathrm{O}_{3}$. Mater Lett 2007, 61: 4759-4762.

[24] Yan M, Lane M, Kannewurf CR, et al. Highly conductive epitaxial $\mathrm{CdO}$ thin films prepared by pulsed laser deposition. Appl Phys Lett 2001, 78: 2342.

[25] Kuznetsova LA, Kuznetsov VL, Rowe DM. Thermoelectric properties and crystal structure of ternary compounds in the $\mathrm{Ge}(\mathrm{Sn}, \mathrm{Pb}) \mathrm{Te}-\mathrm{Bi}_{2} \mathrm{Te}_{3}$ systems. $J$ Phys Chem Solids 2000, 61: 1269-1274.

[26] Jung K-H, Lee KH, Seo W-S, et al. An enhancement of a thermoelectric power factor in a Ga-doped $\mathrm{ZnO}$ system: A chemical compression by enlarged Ga solubility. Appl Phys Lett 2012, 100: 253902.

[27] Mendelsberg RJ, Zhu Y, Anders A. Determining the nonparabolicity factor of the $\mathrm{CdO}$ conduction band using indium doping and the Drude theory. J Phys D: Appl Phys 2012, 45: 425302.

[28] Zhao L-D, Lo S-H, He J, et al. High performance thermoelectrics from earth-abundant materials: Enhanced figure of merit in $\mathrm{PbS}$ by second phase nanostructures. J Am Chem Soc 2011, 133: 20476-20487. 